\title{
Uso das tecnologias na representação descritiva: o padrão de descrição bibliográfica semântica MarcOnt Initiative nos ambientes informacionais digitais
}

\author{
Fabiano Ferreira de Castro \\ Doutorando em ciência da informação (Unesp).Mestre em ciência da informação (Unesp).Grupo de Pesquisa Novas Tecnologias em \\ Informação (Unesp-FFC) \\ E-mail: fabianofcastro@gmail.com
}

Plácida Leopoldina Ventura Amorim da Costa Santos

Doutora em linguística. Professora do Programa de Pós-Graduação em Ciência da Informação (Unesp). Coordenadora do Grupo de Pesquisa Novas Tecnologias em Informação (Unesp-FFC)

E-mail: placida@flash.tv.br

\section{Resumo}

A representação descritiva no panorama atual vem sofrendo mudanças notórias nos modelos de descrição bibliográfica convencionais, permeadas pelas tecnologias de informação e comunicação, o que conduz a ou requer olhares diferenciados no tratamento dos recursos informacionais. Destaca-se que há forte tendência para a disponibilização de conteúdos digitais e falta de uso de padrões adequados para a representação e a descrição desses recursos em ambientes informacionais digitais. A iniciativa MarcOnt surge no bojo da Web semântica, como um padrão de descrição bibliográfica semântica com vistas a representar e descrever recursos informacionais mais exaustivamente e mais bem explicitados para compreensão e entendimento semântico pelas máquinas. Procura-se demonstrar a aplicação da ferramenta MarcOnt em ambiente informacional específico, a Biblioteca Digital Semântica Jerome $L$, para analisar, à luz dos fundamentos da catalogação, as formas de representação dos recursos informacionais. Por meio de uma metodologia de revisão de literatura, objetiva-se identificar e caracterizar o uso e a aplicação do MarcOnt enquanto ferramenta para a construção de formas de representação da informação para bibliotecas digitais e para a Web na atualidade. O MarcOnt apresenta-se como uma iniciativa para os novos modelos de colaboração e compartilhamento de recursos informacionais, na elaboração completa e complexa da descrição bibliográfica, seguindo seu percurso natural sob o auspício da catalogação.

\section{Palavras-chave}

MarcOnt. MARC21. Web Semântica. Bibliotecas digitais. Catalogação automatizada. Padrões de metadados. Informação e Tecnologia.

\section{Use of technology in descriptive representation: the MarcOnt Initiative pattern of semantic-bibliographic description and its impact in digital information environments}

\begin{abstract}
Descriptive representation has changed the conventional models of bibliographic description because of Information and Communication Technologies, demanding different approaches of information resources. There is a strong tendency to offer digital content and a lack of use of proper standards to represent and describe these information resources in digital environments. The MarcOnt initiative emerged from Semantic Web as a standard of bibliographic semantics in order to represent and describe information resources for the semantic interpretation by the machines. This study seeks to demonstrate the application of the MarcOnt tool in a specific information environment - JeromeDL Semantic Digital Library - in order to review according to the foundations of the Cataloging the forms of representing information resources. Through the literature review, this research aims at identifying and characterizing the usage and application of MarcOnt as a tool that builds forms of representing information for digital libraries and the Web. MarcOnt presents itself as an initiative for the new models to share information resources, developing comprehensive and complex bibliographic description, following its natural course under the auspices of the Cataloging.
\end{abstract}

\section{Keywords}

MarcOnt. MARC21. Semantic web. Digital libraries. Automatized cataloging. Metadata patterns. Information and Technology. 


\section{INTRODUÇÃO}

Atualmente, vivencia-se uma mudança de paradigmas, e novos horizontes se abrem à representação descritiva, bem como novas tendências e novos olhares para os registros bibliográficos no século XXI, principalmente no contexto digital, em que novos formatos para a descrição dos recursos informacionais tornam-se imprescindíveis, destacando-se, nessa esfera, o papel dos metadados. O uso intensivo das tecnologias da informação e comunicação (TICs) gera o desenvolvimento de uma gama de padrões de metadados, instrumentos fundamentais nos ambientes de manipulação de dados bibliográficos.

Nesse cenário, Kruk, Decker e Zieborak (2005) destacam o MarcOnt Initiative, que apresenta instrumentos subjacentes à Web semântica, tais como as ontologias e o uso de padrões de metadados, contemplando parâmetros que vão ao encontro dos objetivos das bibliotecas digitais e propiciando meios mais adequados de representar, descrever e organizar os recursos informacionais digitais atrelados aos aspectos semânticos de seus conteúdos.

O presente artigo, por meio de um estudo exploratório e descritivo, traça algumas reflexões sobre as tendências e o uso das tecnologias no campo da representação descritiva, destacando o padrão de descrição bibliográfica semântica MarcOnt e suas imbricações nos ambientes informacionais digitais.

\section{NOVOS OLHARES PARA A DESCRIÇÃO BIBLIOGRÁFICA NO ÂMBITO DIGITAL: A INICIATIVA MARCONT}

Durante as últimas décadas, observou-se que mais e mais informações têm sido publicadas, armazenadas e disponibilizadas na $W e b$. No entanto, somente parte dessas informações, em ambientes específicos como as bibliotecas digitais, possui um padrão de metadados para a descrição bibliográfica que possibilite a padronização dos recursos informacionais para atender aos requisitos da interoperabilidade.

As bibliotecas digitais apresentam-se e atuam na Internet como um segmento em que procuram desenvolver e criar métodos e técnicas para a padronização dos recursos informacionais. Mas garantir/assegurar a interoperabilidade entre "ilhas" de informações bem estruturadas e padronizadas, bem como entre formatos bibliográficos distintos, é uma questão de investigação e preocupação na comunidade científica.

Novas tecnologias baseadas em pesquisas na temática Web semântica desenvolvem-se com o intuito de tentar agregar semântica à descrição bibliográfica para um tratamento mais efetivo dos conteúdos digitais. A $W e b$ semântica oferece algumas soluções para diferentes problemas, criando nova visão para a representação, o armazenamento e o processamento dos dados. Algumas dessas soluções poderiam ser implementadas para potencializar os resultados das buscas no âmbito das bibliotecas digitais.

O projeto MarcOnt Initiative surge, nesse cenário, na perspectiva de tentar solucionar os problemas de descrição e de representação dos recursos informacionais em ambiente digital, como as bibliotecas digitais, por exemplo, atrelado aos padrões de metadados advindos das áreas da biblioteconomia e da ciência da informação, como o MARC21 para a descrição bibliográfica e outros padrões, como o Dublin Core, para a localização de recursos na $W e b$.

MarcOnt Initiative se desenvolveu a partir da Biblioteca Digital Semântica JeromeDL. Como relata Synak (2005), as primeiras experiências na JeromeDL eram positivas, mas logo foram detectados alguns problemas, pois os objetivos iniciais eram desenvolver e aumentar a busca automaticamente, atribuindo semântica aos recursos informacionais, a fim de potencializar os processos de recuperação de informação. As razões pelas falhas iniciais podem ser entendidas de acordo com dois fatos: 
$1^{\circ}$ ) aumentar a busca semanticamente requer a informação sobre os recursos armazenados inicialmente na biblioteca, como descrições semânticas; entretanto, a dificuldade em convencer realmente os bibliotecários de que, criando dados semânticos, resolver-se-ia o problema de trabalhos futuros resultou na construção da primeira biblioteca digital semântica que não utilizava, em suas estruturas, a potencialidade semântica nem em nível mínimo;

$\left.2^{\circ}\right)$ verificou-se que as descrições na JeromeDL poderiam fornecer capacidade de descrição de formatos de metadados conhecidos e usados por comunidades da biblioteconomia, tais como o MARC21. A ontologia simples da JeromeDL, consistindo de somente três conceitos, não foi projetada apenas para esse tipo de dado e metadado (SYNAK, 2005).

Depois de muitos estudos realizados pelos pesquisadores da JeromeDL e a equipe envolvida no projeto, decidiu-se tratar desses problemas criando-se uma ontologia bibliográfica que pudesse contemplar os aspectos semânticos de forma e de conteúdo dos recursos informacionais e que pudesse atuar de forma mais efetiva em uma biblioteca digital semântica. Assim, os requisitos iniciais foram elaborados por Sebastian Ryszard Kruk, e se constituiu o projeto MarcOnt Initiative@2005 .

O que seria o MarcOnt Initiative?

De acordo com Kruk, Synak e Zimmermann (2005a, p. 1),

o objetivo da iniciativa MarcOnt é desenvolver um novo padrão para a descrição bibliográfica na forma de uma ontologia, e relacionar as ferramentas da Web semântica utilizando tecnologias semânticas.

projeto detalhado encontra-se disponibilizado no Portal

Ont no site: Disponível em: <http://portal.marcont.org>. esso em: 10 jan. 2008.
Pode-se afirmar que o MarcOnt Initiative,

seria um padrão de descrição bibliográfica semântica que contempla forma e conteúdo do recurso informacional, a fim de sua identificação e individualização para leitura e processamento compreensíveis por máquina/computador, utilizando-se de instrumentos e ferramentas tecnológicas subjacentes à $W e b$ semântica, podendo ser aplicado no contexto de ambientes informacionais digitais (CASTRO, 2008, p. 123).

O primeiro passo para agregar semântica à descrição das informações em bibliotecas digitais e sua compatibilidade para que ocorra a interoperabilidade é o desenvolvimento de uma ontologia comum. A ontologia pode ser considerada como um instrumento para a descrição bibliográfica dos recursos informacionais, pois se cria uma rede de conceitos com propriedades adequadas e restritas. Kruk, Synak e Zimmerman (2005b, p. 1) alegam que

infelizmente, é muito difícil convencer os bibliotecários a realizarem o esforço da criação da descrição semântica dos recursos, tais como livros, por exemplo.

Pode-se destacar a importância da participação ativa de profissionais de áreas interdisciplinares na criação do MarcOnt, em especial dos bibliotecários, uma vez que esses, em sua formação, possuem as técnicas e habilidades necessárias ao tratamento documentário dos recursos informacionais. A catalogação, enquanto disciplina responsável pela representação descritiva bibliográfica da área, pautada em regras e esquemas de descrição, fornece subsídios para a construção de formas de representação e de descrição padronizadas que possibilitam o armazenamento, a preservação, o uso e o reuso das informações de modo mais eficiente e eficaz.

Um dos motivos desta pesquisa, e na busca por subsídios a fim de caracterizar o MarcOnt, está 
pautado na tese que Synak (2005) procura defender com tanta propriedade, ao mencionar que o MARC21, mesmo sendo um padrão mundial e internacionalmente aceito e adotado pelas bibliotecas e pela comunidade da biblioteconomia, possuindo uma estrutura completa e complexa, não tem um padrão de descrição semântica que contemple a profundidade da descrição, ou ainda, que cubra a extensão requerida ou suportada por ele. Pensando nisso, pode-se considerar que o padrão de descrição bibliográfica semântica MarcOnt pode possibilitar uma melhora na explicitação das informações, permitindo sua compreensão por máquinas nos atendimentos das solicitações de busca realizadas pelo usuário final.

\section{Apresentação da Arquitetura MarcOnt}

Para melhor compreensão do padrão de descrição semântica MarcOnt, os autores Kruk, Synak e Zimmermann (2005) propuseram uma arquitetura inicial, a fim de ter uma visualização de seu funcionamento ao se pensar na questão da interoperabilidade semântica entre padrões de metadados heterogêneos, bem como o acesso aos recursos informacionais numa única interface.

A figura 1 apresenta o delineamento de uma arquitetura inicial dos serviços de mediação MarcOnt.

De acordo com a figura 1, pode-se traçar alguns comentários sobre o funcionamento do padrão de descrição semântica MarcOnt.

A arquitetura forma um fluxo em que a parte superior é a entrada (input), as descrições semânticas estão no centro (MarcOnt ontology), e a parte inferior é a saída (output). Nota-se que o centro da arquitetura é o RDF (Resource Description Framework), considerado o núcleo de armazenamento para descrições semânticas compiladas de outros padrões de metadados.

As caixas na primeira fileira (na entrada) e na última fileira (na saída) da arquitetura representam os formatos inerentes aos tradicionais arquivos neste
FIGURA 1

Arquitetura de serviços de mediação MarcOnt

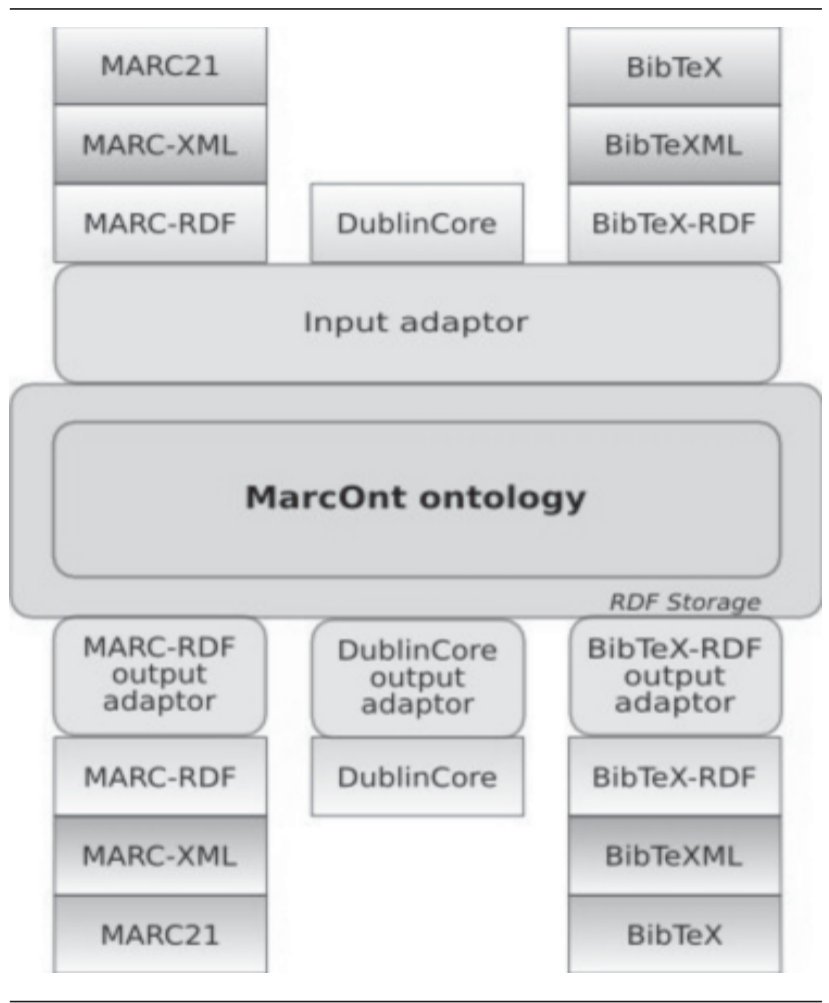

Fonte: Kruk, Synak e Zimmermann (2005a, p. 2).

formato. As caixas no meio (MARCXML, BibTeXML) representam a primeira fase do processo de tradução, em que os arquivos inerentes são analisados gramaticalmente (sintaxe) e seus conteúdos são incluídos no formato XML (Extensible Markup Language).

Os adaptadores de entrada e saída (input e output) são implementados usando-se o mecanismo de inferência Sesame ${ }^{2}$. Do lado dos adaptadores, há as descrições semânticas e, do outro, dados de descrições bibliográficas convertidas para um modelo RDF. Converter diferentes formatos de descrição para o modelo RDF depende do formato

\footnotetext{
${ }^{2}$ Sesame é uma ferramenta, ou seja, um conjunto de classes-base desenvolvido em Java com código aberto para o desenvolvimento de sistemas, com o objetivo de armazenar e consultar dados em RDF. Este sistema é totalmente extensível e configurável no que concerne aos mecanismos de inferência e armazenamento de dados em formato RDF. Maiores informações sobre o sistema Sesame podem ser encontrados no site: Disponível em: <http:// www.openrdf.org/>. Acesso em: 10 mar. 2008.
} 
original do arquivo. Registros em formato MARC21 são armazenados em arquivos binários; então, a conversão exige, primeiro, que tais arquivos sejam transformados para o formato MARCXML e, em fase posterior, transformam-se os dados para o modelo RDF usando XSLT (Extensible Stylesheet Language Transformations).

Synak (2005, p. 75) destaca que ainda não há representações oficiais de dados de MARC21 em RDF, desenvolvido pela Library of Congress (LC), e que os pesquisadores do MarcOnt criaram seu próprio formato (MARCRDF).

Apoiar outros formatos é uma questão de desenvolver adaptadores e conversores apropriados. Atualmente, estamos desenvolvendo adaptadores para MARC21 (SYNAK, 2005, p. 75).

A fase final é traduzir os dados RDF para dentro de estruturas compatíveis com o padrão de descrição bibliográfica MarcOnt. Os estágios precedentes requerem escrever ferramentas simples para que as bibliotecas disponíveis as usem, e possa ser relativamente fácil intercambiá-las (SYNAK, 2005). Assim, pode-se afirmar que essa arquitetura inicial visa ao compartilhamento entre padrões de metadados distintos, podendo proporcionar a interoperabilidade semântica entre bibliotecas digitais, por exemplo, em que as informações semânticas estarão disponíveis e armazenadas em uma só interface, neste caso, no MarcOnt.

Considera-se que o padrão tenha surgido à luz da Web semântica, projetado para atender aos requisitos de interoperabilidade semântica entre ambientes informacionais, como o das bibliotecas digitais. Para garantir a melhora da performance na manipulação de formas de representação bibliográfica e da interação dos sujeitos com o ambiente informacional, tem-se no MarcOnt um ponto de partida para a integração de fontes/ sistemas que se utilizam de padrões de metadados heterogêneos, potencializando a busca e a recuperação dos recursos informacionais, e possibilitando a preservação, o uso e o reuso das informações de maneira mais efetiva, em interface única.

\section{Bibliotecas digitais: modelo de ambientes estruturados e colaborativos}

No contexto digital, pode-se destacar o papel das bibliotecas digitais, que têm muito a contribuir com as questões da padronização da representação dos recursos informacionais, constituindo-se de uma ambiência preestabelecida (metadados) de acordo com regras e normas que permeiam a construção dos recursos, possibilitando maior nível de interoperabilidade entre ambientes informacionais digitais.

Entretanto, com a finalidade de potencializar os serviços que uma biblioteca digital pode oferecer e de acordo com as novas esferas tecnológicas trazidas no bojo da chamada Web semântica, evidenciam-se várias tentativas de melhorar os sistemas de busca e navegação por meio da agregação de tecnologias semânticas aos recursos informacionais, possibilitando aos usuários a obtenção de resultados mais significativos.

Para acompanhar esse cenário de evoluções tecnológicas e como um modelo de aplicação do padrão de descrição semântica MarcOnt, surge a Biblioteca Digital Semântica JeromeDL como uma ambiência que, por meio da semântica, visa a ou procura possibilitar um refinamento nos mecanismos de busca.

A Biblioteca Digital Semântica JeromeDL originouse de experiências e testes da tese de Sebastian Ryzard Kruk na Gdañsk University of Technology (GUT), Polônia, em 2004/2005, tendo contado com a participação e a supervisão da Digital Enterprise Research Institute (DERI), em Galway, na Irlanda, que é uma parte da National University of Ireland, na mesma cidade.

A descrição dos recursos informacionais tem como base a arquitetura de metadados RDF e a realização de buscas semânticas baseadas em ontologias, o 
que significa a possibilidade de melhorias na busca e na usabilidade, com o estabelecimento de maior nível de interoperabilidade entre ambientes informacionais digitais (KRUK; DECKER; ZIEBORAK, 2005).

É oportuno mostrar algumas diferenças no que tange aos aspectos conceituais entre biblioteca digital e biblioteca digital semântica. De acordo com os autores supracitados, pode-se considerar a biblioteca digital semântica aquela que:

- tem a possibilidade de integrar/compartilhar informações baseadas em diferentes padrões de metadados, com anotações semânticas dos recursos e perfil dos usuários;

- pode prover níveis de interoperabilidade não somente com bibliotecas digitais, mas com outros sistemas de informação, como repositórios digitais, com metadados distintos;

- possibilita a construção de interfaces mais amigáveis ao usuário em relação às buscas e à navegação determinadas pela semântica.

- pode apresentar-se como um modelo no que concerne à transição de informações estáticas (somente armazenadas em bibliotecas digitais convencionais) para uma estrutura dinâmica e colaborativa na formação e geração de espaços de construção de conhecimento social (CASTRO, 2008).

Kruk (2006) elucida que as bibliotecas digitais atuais trazem descrições semânticas básicas e que, juntamente com anotações semânticas comunitárias (perfil do usuário) e dos recursos informacionais, podem trazer a próxima ou a nova geração da Internet.

Isso leva a inferir que as bibliotecas digitais se apresentam com uma nova visão, caracterizadas como ambientes sociais colaborativos com o uso e o reuso de informações semânticas, indo ao encontro dos princípios do modelo que vem se constituindo hoje como Web 2.0 (CASTRO, 2008).
Pensando nisso, a Biblioteca Digital Semântica JeromeDL foi projetada a fim de atender desde os profissionais responsáveis pela confecção dos recursos informacionais, até os usuários que irão se beneficiar e utilizar esses recursos.

Para melhor entender o funcionamento da arquitetura da Biblioteca Digital Semântica JeromeDL, bem como a estrutura MarcOnt, descreve-se, a seguir, como ela está disponibilizada para os usuários.

\section{Interfaces de apresentação e representação dos recursos informacionais na Biblioteca Digital Semântica JeromeDL: reflexões sobre representação descritiva e MarcOnt}

Ao se pensar em formas de representação da informação registrada, além da descrição e da representação dos recursos em ambiente informacional, de acordo com normas e padrões que norteiam sua construção, deve-se considerar as formas de apresentação do recurso informacional para favorecer usabilidade aos que se apropriarão das informações no sistema.

As seções seguintes apresentarão de modo sucinto o ambiente da Biblioteca Digital Semântica JeromeDL, apontando como estão construídas as formas de representação dos recursos informacionais e como estão distribuídas para os usuários.

A figura 2, a seguir, mostra a interface de apresentação da biblioteca digital semântica, em que constam alguns links sobre a documentação da biblioteca, as opções de acesso e manuseio aos recursos informacionais, projetos relacionados à biblioteca, os órgãos e instituições que estão vinculados à JeromeDL, breve contextualização da criação da biblioteca entre outros itens.

A figura 3, a seguir, descreve outra interface que contempla as opções de busca aos recursos informacionais, bem como os tipos documentais armazenados na biblioteca digital semântica. Vale destacar que, no menu da biblioteca, tem-se a opção Idiomas, em que o usuário pode escolher em qual 
FIGURA 2

Interface de apresentação da Biblioteca Digital Semântica JeromeDL

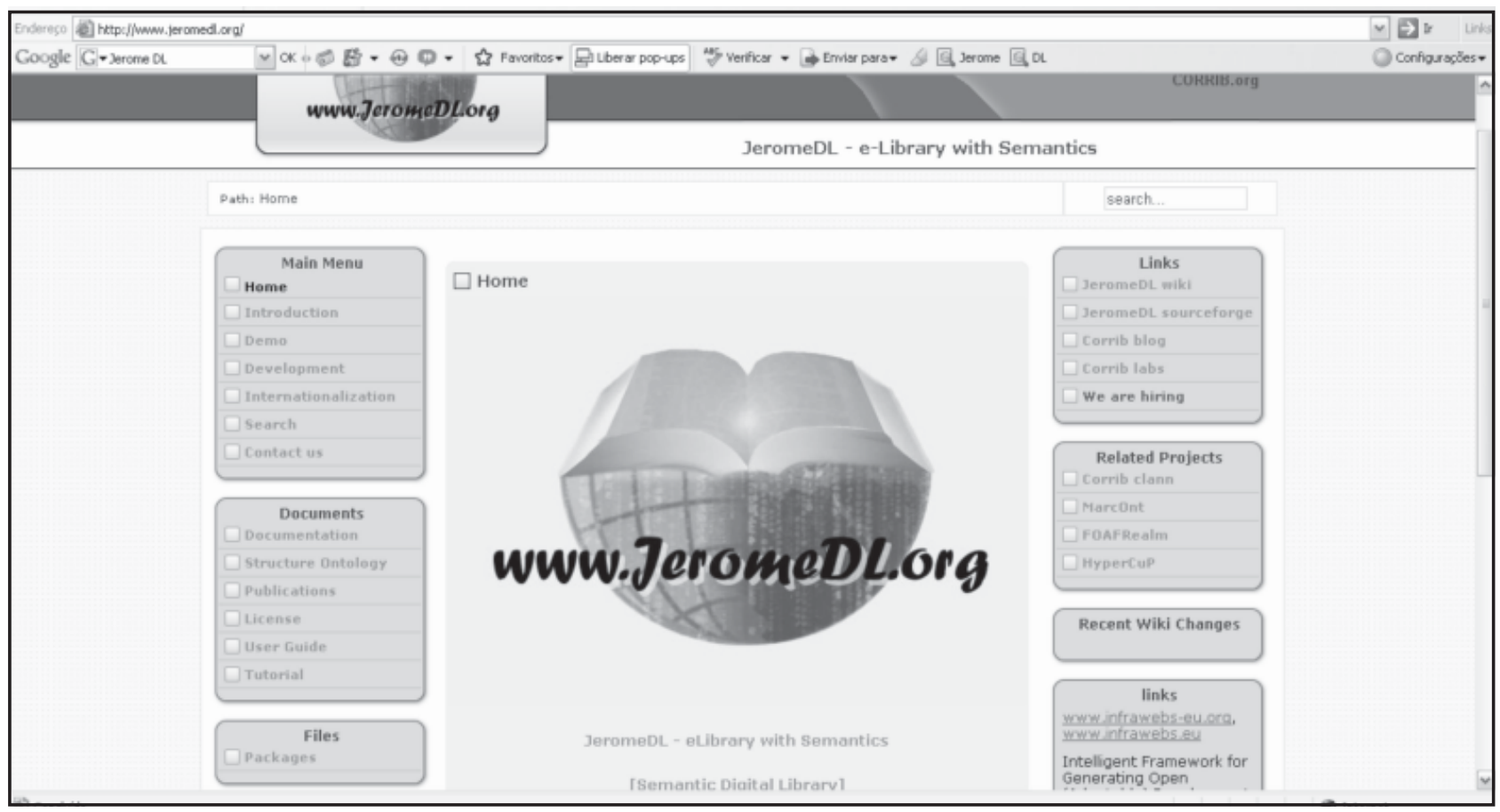

Fonte: disponível em: <http://www.jeromedl.org/>. Acesso em: 31 out. 2007.

\section{FIGURA 3}

Formas de busca e tipos documentais

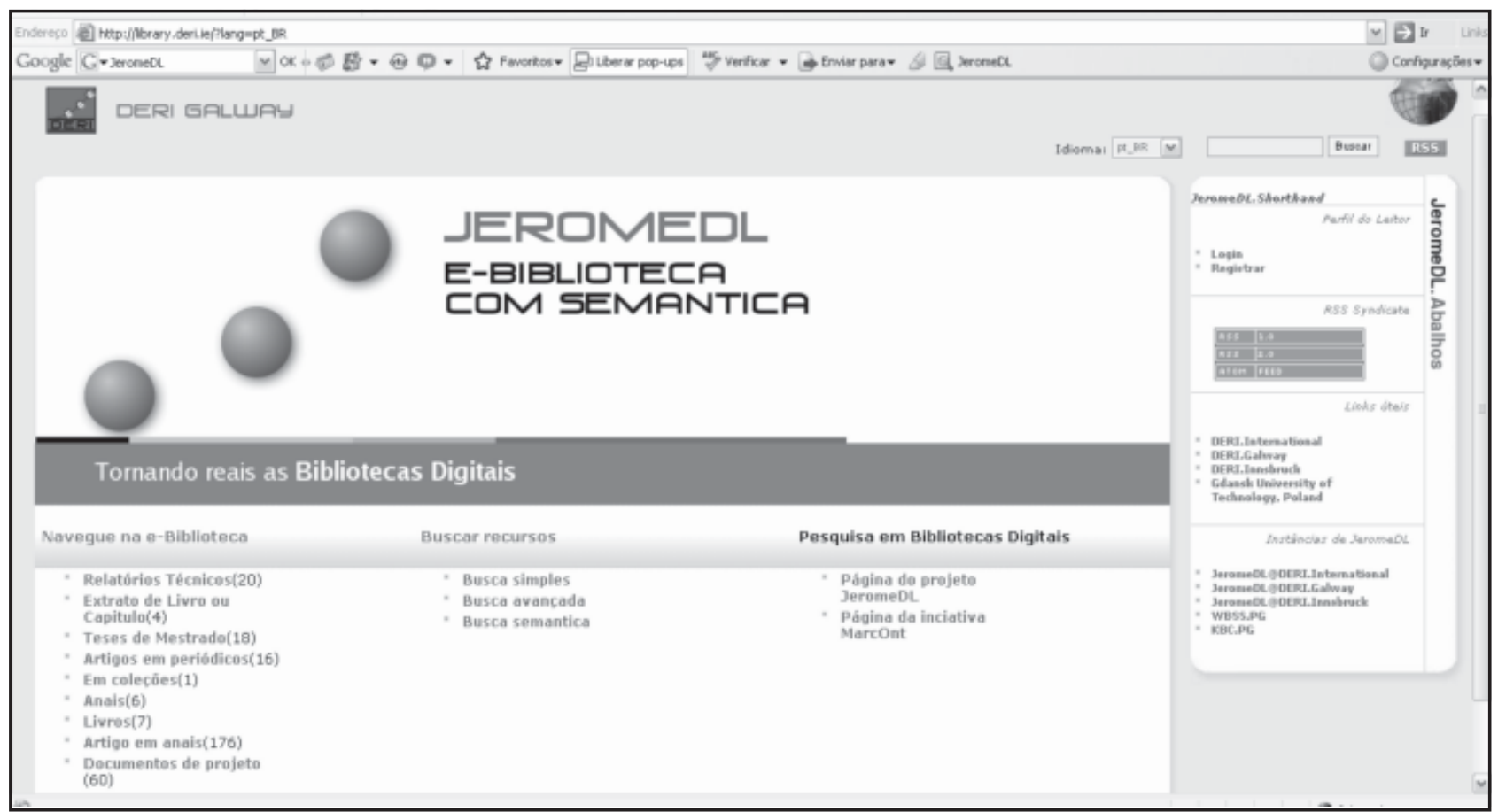

Fonte: disponível em: <http://library.deri.ie/?lang=pt_BR>. Acesso em: 31 out. 2007. 
ele deseja visualizar a interface da biblioteca. Vale destacar que, nesse caso, será usado o idioma português.

Ainda em relação a essa interface, pode-se ressaltar as alternativas de busca aos recursos informacionais, com três opções: simples, avançada e semântica. As buscas simples e avançadas constam de operadores booleanos encontrados em qualquer tipo de biblioteca digital.

Vale atentar para um diferencial na busca avançada em que há opções de se pesquisar pelo conteúdo do recurso informacional e também pelo tipo de padrão de metadado (Dublin Core, BibTeX, MARC21), o que pode ser visto na figura 4.

O grande impacto e o diferencial estão na busca semântica, que se pode visualizar de acordo com a figura 5, a seguir.

O desafio para a Biblioteca Digital Semântica JeromeDL está justamente em fornecer informações semânticas que outras bibliotecas digitais não oferecem. Na opção Select template, o usuário pode escolher entre as seguintes opções: Exiba todos os recursos na biblioteca, Exiba todos os recursos criados por..., Exiba todos os recursos criados por meus amigos, Exiba todos os recursos criados por alunos de... Pode-se dizer que a biblioteca digital semântica funciona como um ambiente colaborativo, conforme mencionado nas seções anteriores, com a participação não somente de profissionais (bibliotecários, informáticos), mas também dos sujeitos (usuários) que se apropriarão dos recursos informacionais.

Destaca-se que a opção de busca semântica não está ativa e que, segundo informações do idealizador da JeromeDL, Sebastian Kruk (2007), encontra-se ainda em fase de experimentos e testes para funcionar em breve.

Em relação aos tipos documentais que estão armazenados na Biblioteca Digital Semântica JeromeDL, escolheu-se um recurso do tipo livro para análise sob o prisma da catalogação: como o recurso estaria representado para o usuário.

\section{FIGURA 4}

\section{Interface de busca avançada comentada pelo autor}

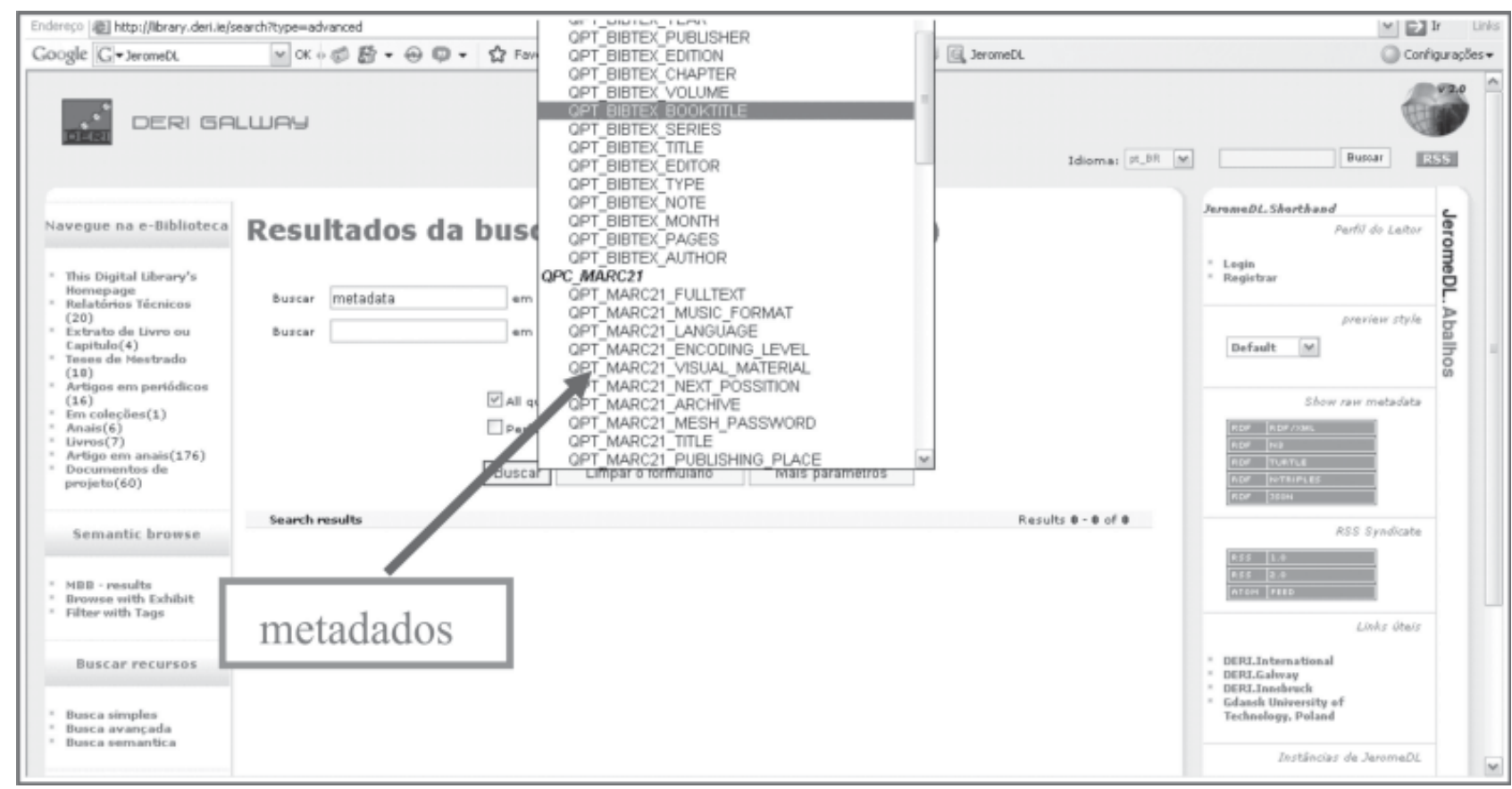

Fonte: disponível em: <http://library.deri.ie/search?type=advanced>. Acesso em: 31 out. 2007. 


\section{FIGURA 5}

\section{Interface de busca semântica}

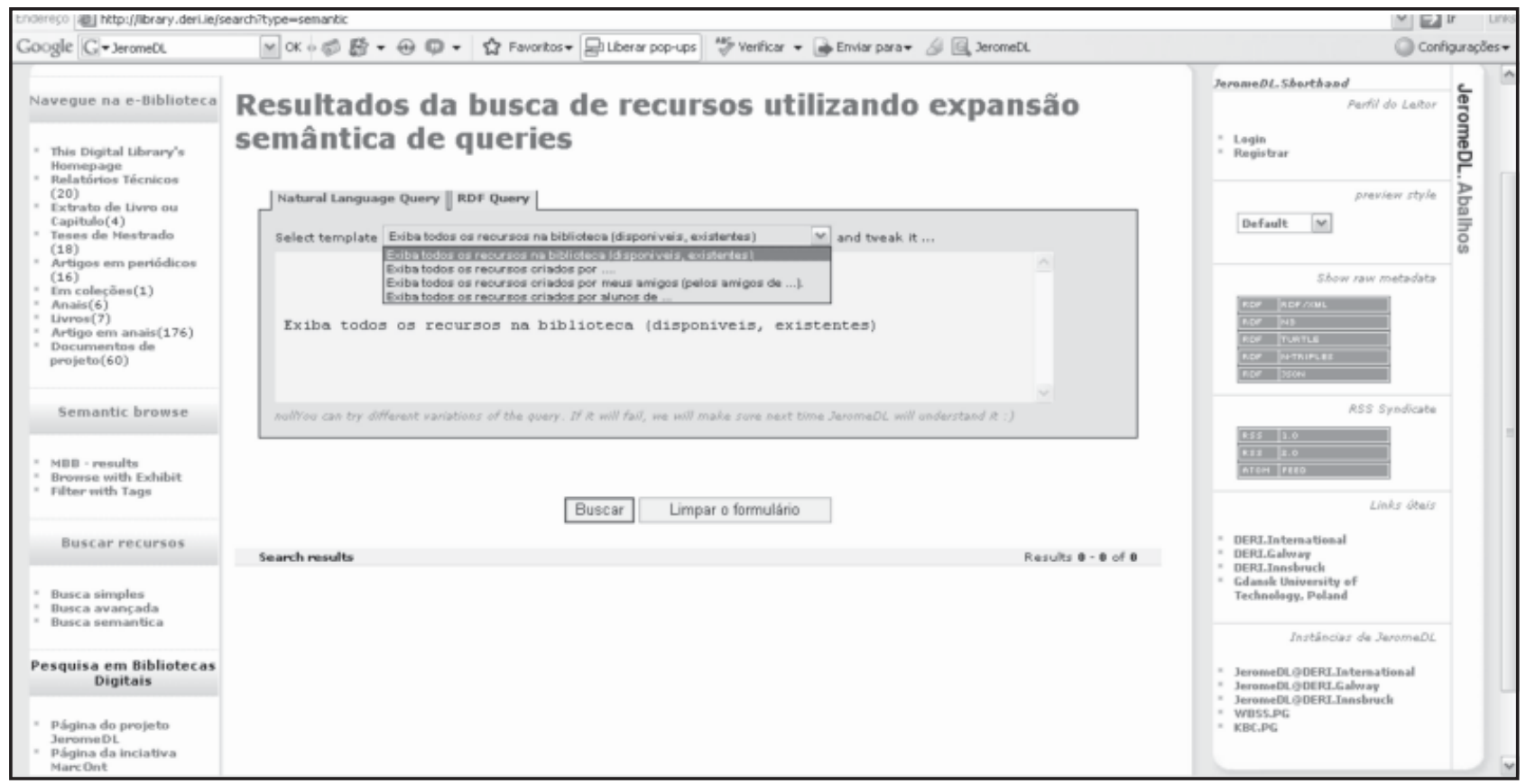

Fonte: disponível em: <http://library.deri.ie/search?type=semantic>. Acesso em 31 out. 2007.

A figura 6 apresenta algumas informações do recurso, como, por exemplo, as palavras-chave, descrições adicionais do recurso e uma opção de apresentação do recurso, de acordo com um padrão de metadados em uma só interface.

\section{FIGURA 6}

\section{Recurso informacional livro}

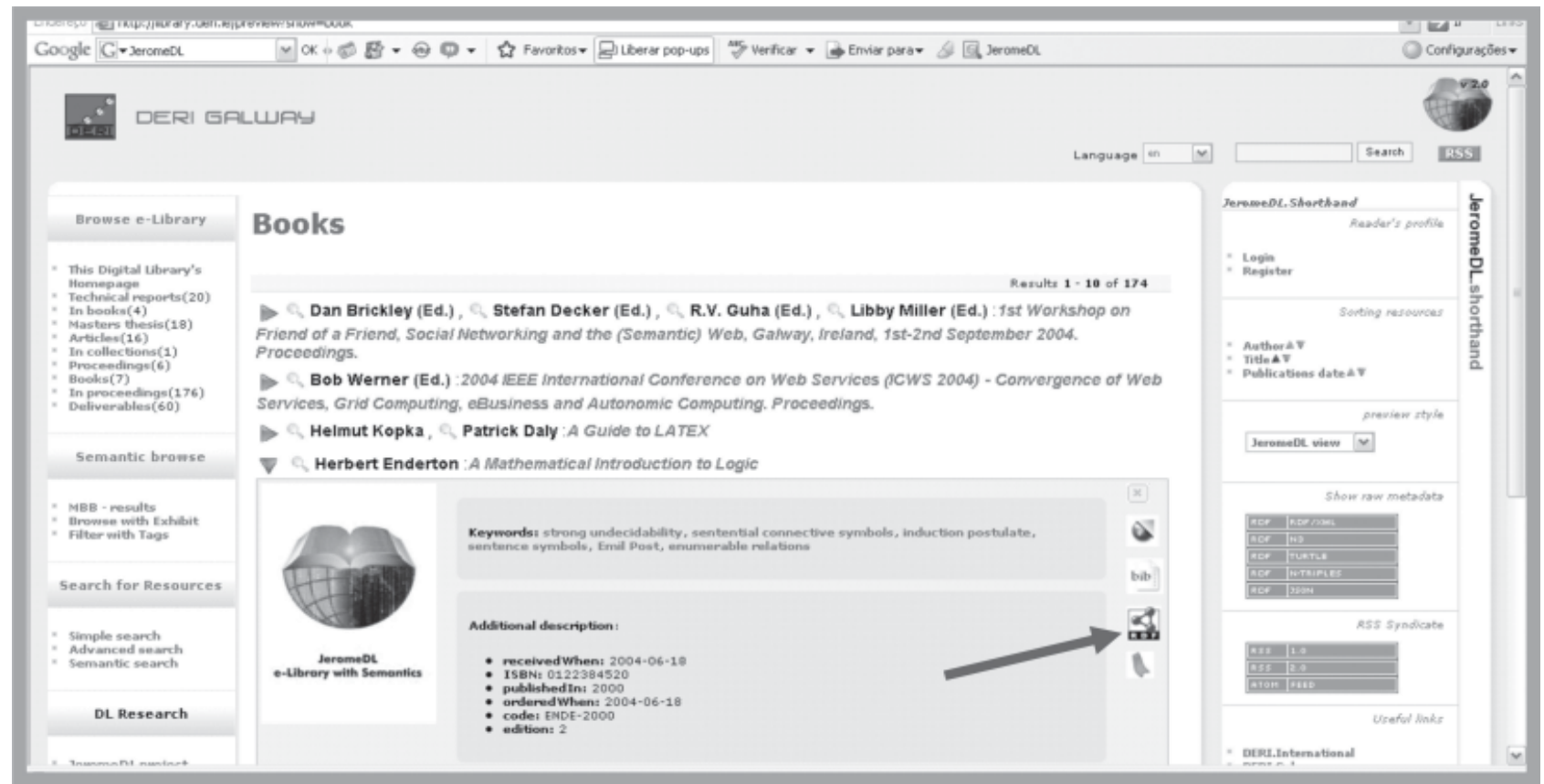

Fonte: disponível em: <http://library.deri.ie/preview?show=book>. Acesso em 31 out. 2007. 
Além disso, a Biblioteca Digital Semântica JeromeDL possibilita a apresentação dos recursos informacionais no momento da busca e sua visualização em um padrão de metadado de acordo com a preferência do usuário, conforme pode ser visto na figura 7 .

A figura 7 mostra um recurso informacional apresentado ao usuário numa única plataforma contemplando os padrões de metadados vislumbrados na iniciativa MarcOnt (BibTeX, BibTeXML, BibTeXRDF, Dublin Core, MARC21, MARCXML, MARCRDF e MarcOnt), uma vez que existe nessa interface a possibilidade de interoperabilidade entre padrões de metadados heterogêneos.

A figura 8, a seguir, mostra a representação de um recurso informacional no padrão de descrição bibliográfica semântica MarcOnt.

De acordo com a figura 8 , pode-se considerar que o MarcOnt utiliza para o armazenamento das suas descrições a arquitetura de metadados RDF, possibilitando um nível de interoperabilidade entre os campos do registro, bem como a possibilidade de compartilhamento entre outras bibliotecas digitais e sistemas de informação que fazem uso de padrões de metadados heterogêneos.

Faz-se necessário apresentar alguns comentários pautados e vivenciados na prática biblioteconômica, mais especificamente à luz dos fundamentos da catalogação, que permite a padronização da descrição e da representação dos recursos informacionais.

Um dos pontos que vale a pena ser destacado é como se apresenta uma descrição em MARC21, por exemplo, na Biblioteca Digital Semântica JeromeDL. Em outras buscas realizadas na biblioteca com a finalidade de verificar a representação e a descrição de um recurso informacional, foram observadas algumas falhas nesses quesitos. Foi encontrado um registro MARC21 que possuía mais de um autor responsável pela obra. E o mais curioso era que o campo 100 (Entrada Principal - Nome Pessoal) do registro estava repetido. De acordo com

\section{FIGURA 7}

Formas de apresentação dos recursos informacionais

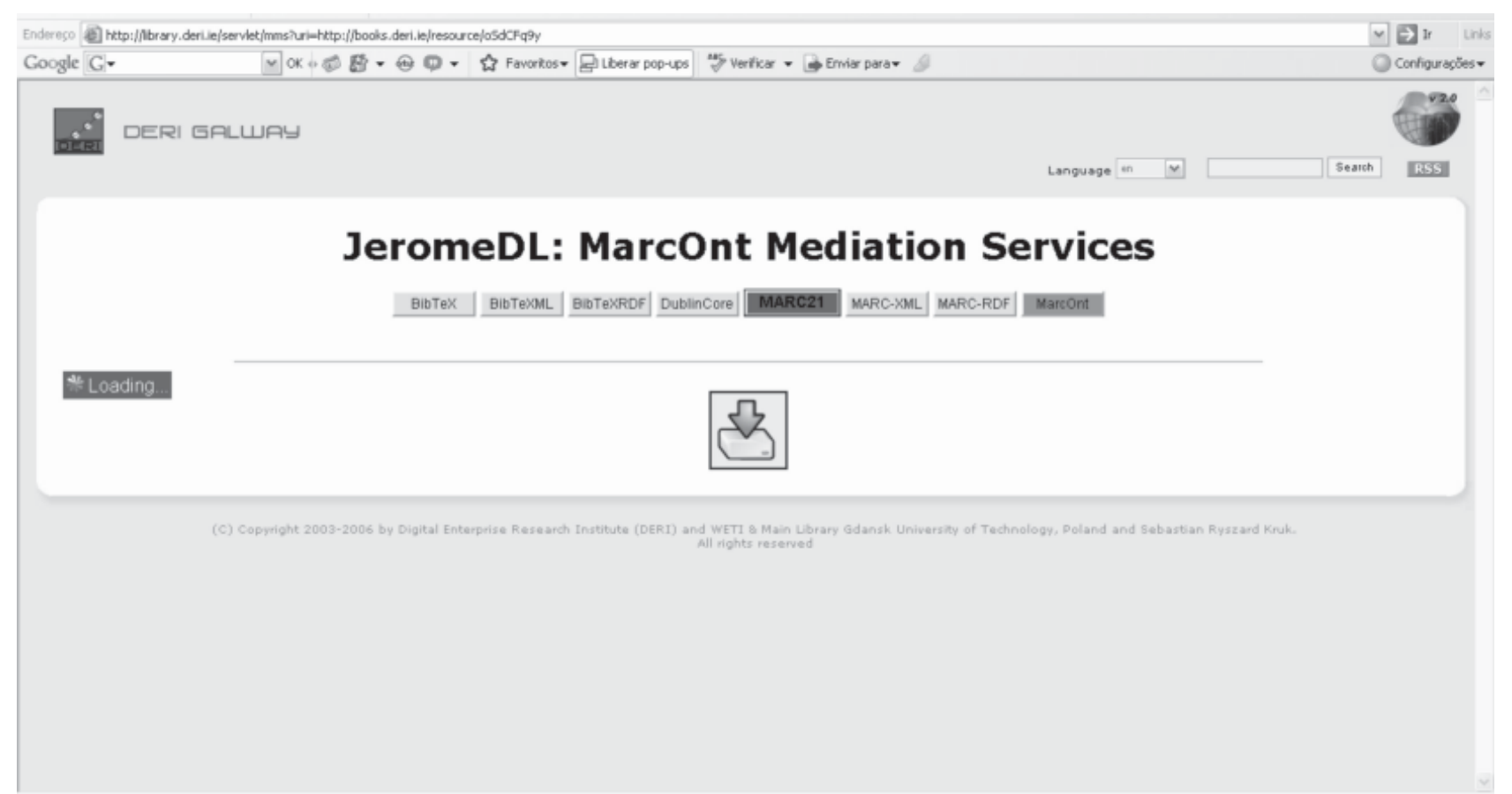

Fonte: disponível em: <http://library.deri.ie/servlet/mms?uri=http://books.deri.ie/resource/oSdCFq9y>. Acesso em 31 out. 2007 
FIGURA 8

Representação de um recurso informacional em MarcOnt

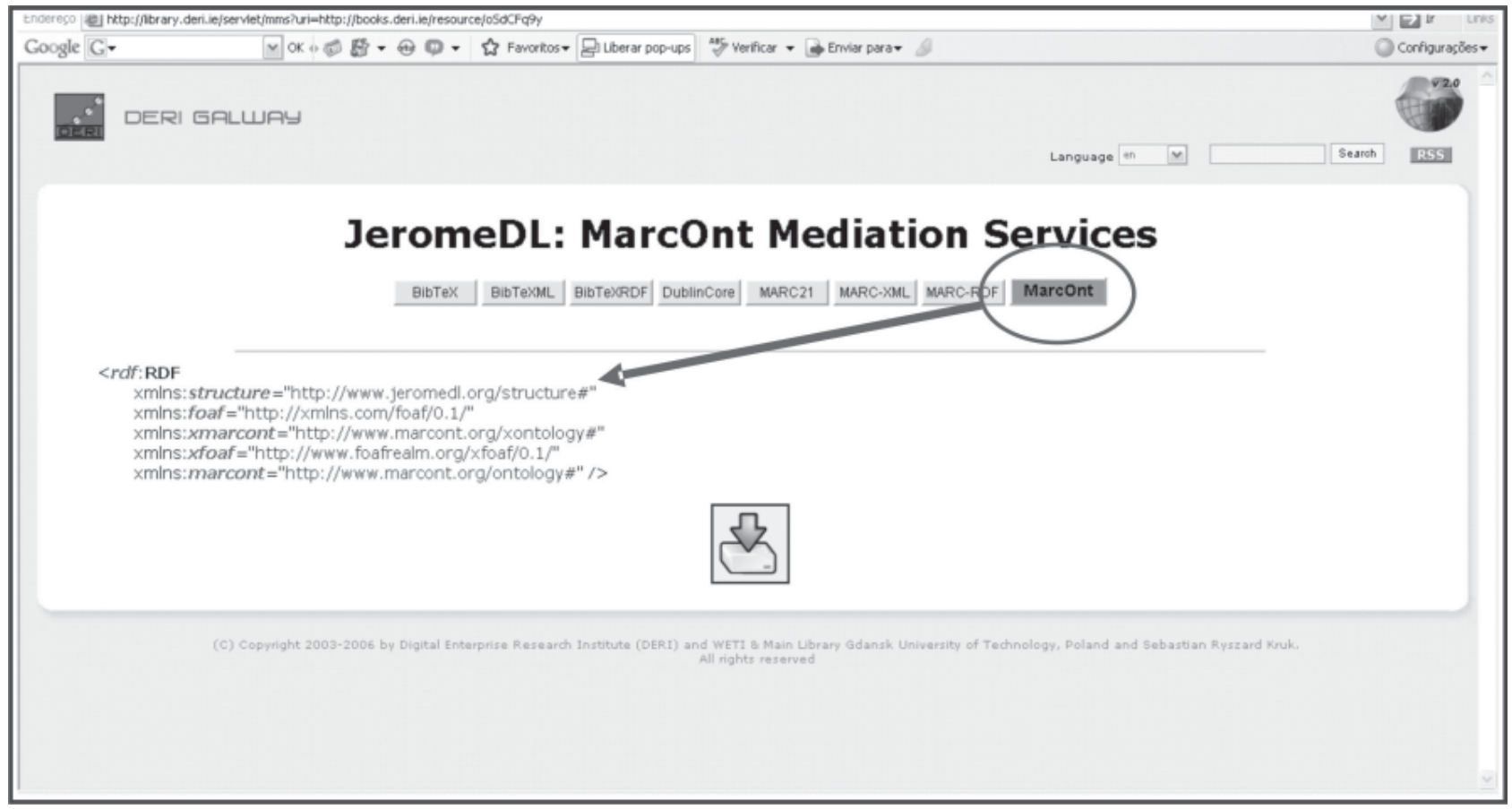

Fonte: disponível em: < http://library.deri.ie/servlet/mms?uri=http://books.deri.ie/resource/oSdCFq9y>. Acesso em 31 out. 2007.

a estrutura MARC, este campo não se repete, pois, conforme os esquemas de descrição AACR2 (Código de Catalogação Anglo Americano - 2a . ed.), que anteveem ou norteiam a construção do campo numa estrutura legível por máquina MARC, seria necessária a criação de uma entrada secundária de autoria, que no registro MARC é dada pelo campo 700 (Entrada Secundária - Nome Pessoal). Vale relembrar também que, até o presente momento, não se encontram disponíveis representação e descrição em MARC21 na sua totalidade, julgando-se relevantes, segundo Kruk (2006), apenas os campos de autoria (100) e título (245) para a busca e a recuperação dos recursos informacionais.

Dessa forma, o bibliotecário é considerado protagonista na construção de regras para o estabelecimento da semântica para outros campos do registro MARC21, podendo estes compartilhar informações semânticas não só no mesmo registro/ estrutura, mas com outros padrões de metadados e em sistemas diferentes.
No que tange à melhora na performance de interoperabilidade entre os padrões de metadados, vale salientar que MarcOnt poderá oferecer regras que permitirão às máquinas/computadores a leitura e o entendimento dos campos de um registro bibliográfico; por exemplo, no campo 245 do padrão de metadados MARC21 e de acordo com os esquemas de descrição do AACR2, há diferentes tipos de títulos, como principal, uniforme, equivalente e outros. Atualmente, sabe-se que a máquina/computador não consegue fazer a diferenciação entre títulos, apenas identifica que 245 significa título. O padrão de descrição bibliográfica semântica MarcOnt visa, por meio da semântica dos conteúdos, a oportunizar uma compreensão explícita das variações de significados dos títulos, no intuito de proporcionar maior interoperabilidade entre padrões de metadados heterogêneos e, consequentemente, com a adoção e o uso do MarcOnt, o estabelecimento da interoperabilidade semântica entre ambientes e sistemas informacionais distintos. 


\section{À GUISA DE CONSIDERAÇÕES}

As tecnologias da informação e comunicação (TICs) influenciam a construção de formas de representação da informação registrada, tomando como modelo de aplicação as bibliotecas, sejam essas convencionais ou digitais, que se apresentam como redes informacionais estruturadas com modelos de ambientes colaborativos.

A catalogação, enquanto disciplina que orienta a descrição na biblioteconomia, tem papel decisivo na manipulação de formas de representação bibliográficas e da interação de sujeitos psicossociais com o ambiente informacional.

A relação entre o padrão de metadados MARC21 e o MarcOnt não está na transformação da estrutura preestabelecida do MARC21 para a linguagem da ontologia, mas no fornecimento de informação semântica à descrição dos recursos, de modo a favorecer uma compreensão pela máquina/ computador.

Os Requisitos Funcionais para Registros Bibliográficos (FRBR), os padrões de metadados já consagrados na área de biblioteconomia, como MARC21, juntamente com o padrão de descrição bibliográfica semântica MarcOnt, atuam em sinergia, pois podem possibilitar maior potencialidade nos relacionamentos entre registros bibliográficos e a amplitude nas solicitações de busca pelo usuário final.

O padrão de descrição bibliográfica semântica MarcOnt é um instrumento tecnológico que, no contexto das bibliotecas digitais e da Web de modo geral, apresenta-se como ferramenta capaz de oportunizar, ao tratamento dado aos recursos informacionais, uma descrição de forma e de conteúdo legível por máquinas, com a possibilidade de resultados compreensíveis aos humanos para a apresentação das informações com vistas a uma recuperação mais eficiente, além da garantia da apresentação das informações para o uso, a preservação e o reuso para os usuários.
Para o funcionamento pleno do padrão de descrição bibliográfica semântica MarcOnt, faz-se necessário que ele compreenda e absorva a égide dos conceitos oriundos da catalogação, uma vez que esta proporcionará e subsidiará os elementos fundamentais para a construção padronizada da representação.

\section{AGRADECIMENTOS}

À Coordenação de Aperfeiçoamento de Pessoal de Nível Superior (Capes), pelo suporte financeiro à pesquisa.

Artigo submetido em 06/12/2008 e aceito em 14/05/2009.

\section{REFERÊNCIAS}

CASTRO, F. F. de. Padrões de representação e descrição de recursos informacionais em bibliotecas digitais na perspectiva da ciência da informação: uma abordagem do MarcOnt initiative na era da web semântica. 2008. 201 f. Dissertação (Mestrado em Ciência da Informação) Faculdade de Filosofia e Ciências, Universidade Estadual Paulista, Marília, 2008.

KRUK, S. R. JeromeDL 2.0: a digital library on social semantic information spaces. [2006]. Disponível em: <http:// www.sebastiankruk.com/storage/presentation/ jeromedl2.0stable_gut/img0.html>. Acesso em: 25 ago. 2007.

. The role of ontologies in semantic digital libraries. In: NKOS WORKSHOP, 1. [2007], Alicante, Spain. Electronic proceedings... Alicante, Spain: [s. n.], [2007]. p. 30. Disponível em: <http://www.sebastiankruk.com/storage/presentation/ nkos2006/img0.html>. Acesso em: 25 ago. 2007.

; DECKER, S.; ZIEBORAK, L. JeromeDL: reconnecting digital libraries and the semantic web. Chiba, Japan: [s. n.], 2005. Disponível em: <http://www.marcont.org/marcont/pdf/ www2005_jeromedl.pdf $>$. Acesso em: 14 jan. 2006.

; SYNAK, M.; ZIMMERMANN, K. Maront initiative: mediation services for digital libraries. 2005a. Disponível em: <http://www.marcont.org/marcont/pdf/marcontecdl2005.pdf >. Acesso em: 16 jan. 2006.

MarcOnt initiative: integration ontology for bibliographic description formats. Madrid: [s. n.], 2005b. Disponível em: <http:/ / d c 2005 . uc $3 \mathrm{~m}$. es / program / presentations / Thursday $\% 2015 . \% 2015.30 \% 20$ h\%20-\%20s.kruk.pdf>. Acesso em: 16 jan. 2006.

SYNAK, M. MarcOnt ontology: semantic MARC21 description for L2L \& L2C communication. 2005. 126 f. Master's Thesis (Informatics, Distributed Computer Systems)- Faculty of Eletronics, Telecommunications and Informatics, Gdañsk University of Technology, Gdañsk, 2005. Disponível em: <http:// library.deri.ie/servlet/showPDF?docId $=$ http $\% 3 a \% 2 f \% 2$ flibrary. deri.ie $\% 2$ fresource $\% 2 \mathrm{f} 3 \mathrm{a} 9 \mathrm{faf} 28 \&$ chapter $=1 \&$ view $=\mathrm{pdf}>$. Acesso em: 25 jul. 2007. 